\title{
Ruptura amorosa, proceso del duelo y aspectos académicos en estudiantes universitarios
}

\section{Love breakup, grieving process and academic aspects in university students}

\author{
Diana B. Mendoza-Guerrero ${ }^{a}$, Rebeca M. Guzmán-Saldaña ${ }^{b}$, Abel Lerma-Talamantes ${ }^{c}$, Lilián \\ E. Bosques-Brugada ${ }^{d}$
}

\begin{abstract}
:
The factors associated with the break-up of a couple are in some cases difficult to overcome, bringing with them important consequences in different spheres of life of the people who experience them. The aim of this study was to explore in university students how the love breakup occurs (in the last six months), and to observe the course that the grieving process follows, as well as to analyze its possible relationship with academic aspects. A non-experimental design was followed, with a qualitative type of exploratory study. $\mathrm{N}=6$ university students participated voluntarily, who participated in a focus group interview. The results showed that most experienced the love breakup as an experience linked to the mourning of the loss, which generated painful feelings and thoughts, which affected different areas of their life, which had as main causes the breakup to infidelity and lack of communication; which also brought with it academic consequences, such as low grades, failed subjects, failure to perform tasks, feeling tired and lack of concentration and motivation, among others. It is important to intervene from the universities in a preventive way during the grieving process due to the break-up with the partner, in order to avoid, on the one hand, the triggering of a complicated clinical picture linked to depression; and on the other hand, to avoid the drop-out, the failure, and even the possible drop-out.
\end{abstract}

Keywords:

Love Breakup, Couple, Perception of the Dueling Process, Academic Aspects, University

\section{Resumen:}

Los factores asociados al rompimiento de una pareja en algunos casos son difíciles de superar, trayendo consigo consecuencias importantes en diferentes esferas de la vida de las personas que los experimentan. El objetivo de este estudio fue explorar en estudiantes universitarios cómo se presenta la ruptura amorosa (en los últimos seis meses), y observar el curso que sigue el proceso de duelo, del mismo modo analizar su posible relación con aspectos académicos. Se siguió un diseño no experimental, con un tipo de estudio exploratorio, de corte cualitativo. Participaron de manera voluntaria $n=6$ estudiantes universitarios, que realizaron una entrevista con formato de grupo focal. Los resultados evidenciaron que la mayoría vivió la ruptura amorosa como una experiencia vinculada al duelo de la pérdida, que generó sentimientos y pensamientos dolorosos, que afectó diferentes áreas de su vida, que tuvo como causas principales del rompimiento a la infidelidad y la falta de comunicación; que además trajo consigo consecuencias de tipo académico, tales como bajo promedio de calificaciones, materias reprobadas, incumplimiento en tareas, sensación de cansancio y falta de concentración y motivación, entre otras. Es importante intervenir desde las universidades de una manera preventiva durante el proceso de duelo por el rompimiento con la pareja, para evitar por un lado, que se desencadene un cuadro clínico complicado vinculado a la depresión; y por otra parte, evitar la baja en las calificaciones, la reprobación, e incluso la posible deserción escolar.

\section{Palabras Clave:}

Ruptura Amorosa, Pareja, Percepción del Proceso de Duelo, Aspectos Académicos, Universitarios

\section{Introducción}

Las relaciones de pareja corresponden a una de las uniones más intensas que pueden establecerse entre los

\footnotetext{
${ }^{a}$ Autora de Correspondencia, Universidad Autónoma del Estado de Hidalgo, email: me306492@uaeh.edu.mx

b Universidad Autónoma del Estado de Hidalgo, https://orcid.org/ 0000-0003-0877-4871, email: rguzman@uaeh.edu.mx

c Universidad Autónoma del Estado de Hidalgo, https://orcid.org/ 0000-0001-7212-641X, email: abel_lerma@uaeh.edu.mx

d Universidad Autónoma del Estado de Hidalgo, https://orcid.org/ 0000-0002-3969-683X, email: lilian_bosques@uaeh.edu.mx
} 
seres humanos, y son el vínculo de mayor significación fuera de la familia de origen. ${ }^{1}$

El comienzo de una relación emocional con la pareja es el preludio de una etapa, que corresponde a un salto importante en el desarrollo de la propia relación y que además será parte de modificaciones en el propio mundo donde el desarrollo personal será enriquecido por nuevas vivencias que pueden marcar vínculos relevantes. ${ }^{2}$ Pineda señaló que cuando se consolida una relación y se crea un proyecto de vida en común, ambos miembros de la pareja deben encarar cambios importantes en su estilo de vida. ${ }^{3}$

La relación de pareja es fundamental para el ser humano y la ruptura de esta puede ser un suceso que desencadene un duelo con efectos en la salud física y mental de la persona. ${ }^{4}$

El duelo se concibe como un proceso con un inicio y un final claramente definido; es un estado en el cual la persona comunica o desarrolla una consecuente reacción que tiene implicaciones psicológicas ante una pérdida ${ }^{5,6}$. Al respecto se han definido dos distintas y bien diferenciadas categorías sobre el duelo, la primera de ellas contempla el duelo por el fallecimiento de una persona con la que se mantuvo una relación de apego, y la otra es el duelo debido a la pérdida de algún objeto material o situación que incluye la ruptura de una relación sentimental. ${ }^{5}$

Algunos investigadores han descrito el proceso del duelo a partir de etapas, la mayoría de ellos coinciden en definir cada una de estas considerando que no se presentan de manera lineal, ya que puede haber avances y retrocesos. Por ejemplo, Kübler-Ross señala en primer lugar a la "Negación" en la cual la persona niega la pérdida del ser, considerando que es un mecanismo de protección para sobrevivir a la pérdida, le sigue la "Ira", que puede manifestarse hacia la persona que ya no está, o se dirige hacia sí mismo por sentir que no hizo nada para prevenir la separación, en tercer lugar está la "Negociación" que se refiere a momentos en que la persona reflexiona sobre lo sucedido e intenta buscar alguna explicación, reflexiona acerca de su futuro y de su vida; esta etapa se acompaña de sentimientos de culpa, en la "Depresión" la persona se concentra en el presente y su realidad de pérdida, lo que genera que experimente sentimientos de tristeza y finalmente la "Aceptación" de la pérdida del ser y esta realidad es permanente. ${ }^{7}$

Particularmente, en la ruptura de una relación amorosa el duelo implica -en la mayoría de los casos- un desequilibrio emocional, presentándose sentimientos vinculados con enojo, tristeza, confusión, y también consecuencias en la salud física. $^{8}$

Sánchez Aragón ${ }^{9}$, describió cuatro etapas del duelo amoroso caracterizadas por reacciones diversas claramente enmarcadas dentro de aspectos como: la negociación (negación y búsqueda de alternativas para regresar con la pareja), la hostilidad (la persona se muestra agresiva, desorganizada y decaída emocionalmente al no tener una solución para continuar con la relación), la desesperanza (pesimismo, depresión y decepción), y la seudoaceptación (expresión de hostilidad hacia la ex pareja, con intentos de reorganización y de aceptación del rompimiento y reconocimiento del motivo por el que no se ha podido establecer una negociación de reconciliación).

Córdova ${ }^{10}$, describe cinco razones por las cuales una relación sentimental termina: a) Desconexión y desinterés que puede originarse por parte de ambos o por uno de los miembros de la relación, que presenta una falta de interés o una disminución en la emoción de estar con el otro, y como consecuencia una reducción en la pasión de la relación. b) No cumplir con las expectativas, esto puede provocar un sentimiento constante de frustración y fracaso al esperar una serie de cambios o mejoras del otro en un determinado tiempo. c) Sensación de "no va a cambiar", como resultado de frecuentes peleas o desacuerdos que no se resolvieron; esto puede avanzar al punto de perder el interés y dar fin a la relación. d) Aparición de terceros, en este sentido no necesariamente debe darse por una infidelidad, incluye el constante involucramiento de amigos, familiares, compañeros a los que se les otorga más importancia que a la relación. e) Confianza, si durante la relación surgen mentiras o reserva de información, es muy posible que comiencen a salir dudas en otros aspectos que la pareja diga o haga.

Una ruptura de pareja es una experiencia muy estresante y difícil emocionalmente, que implica entre otros aspectos la pérdida de proyectos de vida ${ }^{4}$. Perder al ser amado acarrea sentimientos de dolor, desesperación y enojo ante la decepción de afrontar una realidad en la cual las expectativas que habían sido colocadas en la expareja no resultaron posibles ${ }^{11}$. De acuerdo con los hallazgos de Fisher $^{12}$, existe un gran número de casos de ansiedad, depresión y suicidios ocasionados por el dolor de una ruptura amorosa.

Ahora bien, no solo desde el punto de vista emocional la ruptura amorosa ocasiona importantes consecuencias, también se han observado problemáticas asociadas con el desempeño académico, particularmente en adolescentes y jóvenes estudiantes. Por ejemplo, Medina, Fereira, y Marzol $^{13}$, en universitarios venezolanos que recursaban una asignatura encontraron que el $16.5 \%$ de ellos estaban pasando por una ruptura amorosa, y que esta situación impactaba de manera importante en su rendimiento académico. Pulido Navia y Lubo Carbajal ${ }^{14}$ en universitarios colombianos identificaron que el empeño y la atención en su desenvolvimiento académico se entorpecía debido a la presencia de pensamientos vinculados a rupturas amorosas, que incluían 
manifestaciones verbales de profunda tristeza, sensaciones de desánimo al momento de levantarse por las mañanas, el no deseo de salir de casa, la inasistencia a algunas clases, así como falta de concentración adecuada.

La depresión también llega a manifestarse ante la pérdida de la relación, un reporte realizado por Morán ${ }^{15}$ en México en estudiantes universitarios, evidenció que ciertas actitudes frente a una ruptura son comunes, tales como altos niveles de tristeza y ansiedad, pérdida de interés o placer, sentimiento de culpa, falta de autoestima, sensación de cansancio, falta de concentración, llanto, problemas alimentarios, desmotivación, aislamiento; así mismo, se afecta el desempeño en el trabajo, en las actividades sociales, y/o académicas. Alvarado Ruiz y Gómez Ramírez ${ }^{16}$, en un estudio con universitarios también mexicanos encontraron que estos se sentían afectados de manera muy importante cuando discutían con su pareja, y como consecuencia manifestaban "no sentir las ganas de siempre", incluía el estar triste y el no lograr concentrarse. Los autores del estudio señalan la relevancia de tener una estabilidad psicosocial, ya que ésta puede impactar en su desempeño académico.

Ahora bien el efecto académico de una ruptura amorosa también impacta en el resultado final de algunas asignaturas, llegando incluso a ser la causa de calificaciones reprobatorias. En un reporte hecho por Martínez-Sánchez, Hernández-Arzola, Carillo-Méndez y Hernández Miguel 17, realizado en universitarios mexicanos, se encontró que durante un periodo escolar de un total de 1133 alumnos, el 38.4\% habían reprobado alguna materia en el desarrollo de su vida estudiantil universitaria, y al analizar los factores asociados, el aspecto emocional vinculado a la ruptura amorosa cobró relevancia en un $17.9 \%$.

Así mismo, en un estudio desarrollado en México con 90 jóvenes universitarios a los cuales se les aplicó una red semántica, se encontró que ante el estímulo "ruptura de una relación de pareja significativa", las palabras asociadas presentaban sentimientos y emociones negativas, y con mayor frecuencia palabras como: tristeza, dolor, enojo, engaños e infidelidad; cabe señalar, que también se manifestaron 20 palabras positivas, entre las que destacan: aceptación, aprendizaje, recuperación, crecimiento, entre otras. Esto indica que probablemente se requiere atravesar por un proceso de duelo para lograr resultados positivos ${ }^{12}$. Es importante considerar en este sentido, que no todo en el duelo es "negro", Bustos Caro ${ }^{11}$ describe efectos constructivos para las personas que lo han padecido, y una vez que lo superan logran nuevos significados que favorecen el crecimiento personal, y afrontan nuevos retos con mayor seguridad, considerando que la separación demanda la construcción de una nueva identidad y estilo de vida, así como la restructuración de las relaciones interpersonales ${ }^{18}$.

El rompimiento de pareja en algunos casos implica un duelo, la importancia de estudiar esta problemática representa un reto no solo para quien lo experimenta, sino también para quien en su campo profesional busca ayudar o apoyar a quien lo padece. La importancia de abordar aspectos académicos y su relación con la ruptura amorosa en estudiantes del nivel superior lleva a reconocer varias aristas para su comprensión, haciendo énfasis como en este caso, en aspectos del desarrollo de la vida de pareja de los jóvenes, así como el duelo que pudiera estarse viviendo, y cómo esto afecta en sus calificaciones escolares. Este planteamiento da origen al objetivo de la presente investigación, que consiste en explorar los posibles efectos académicos que puedan presentarse durante el proceso de duelo ante el rompimiento de pareja, mediante información obtenida de vivencias de estudiantes universitarios.

\section{Método}

\section{Participantes}

A través de un muestreo no probabilístico intencional, se seleccionó a $\mathrm{N}=6$ participantes voluntarios que atravesaban por una ruptura amorosa, y que tenían de cero a seis meses de haber finalizado la relación. Fueron cinco mujeres y un hombre, con una $\bar{x}=22$ años de edad, estudiantes regulares de distintas licenciaturas (ver tabla 1).

Tabla 1.

Datos de los participantes

\begin{tabular}{lc}
\hline Licenciatura que estudian & $\mathrm{n}$ \\
\hline Ciencias computacionales & 1 \\
Contaduría & 1 \\
Nutrición & 1 \\
Psicología & 3 \\
Tiempo de haber terminado & \\
la relación & $\mathrm{n}$ \\
(en meses) & \\
0-3 meses & 2 \\
3-5 meses & 3 \\
5-6 meses & 1 \\
\hline
\end{tabular}

Tipo de Diseño

El diseño utilizado fue no experimental, transversal

\section{Tipo de Estudio}

En estudio fue exploratorio 


\section{Técnica de Investigación}

Para recabar información, se utilizó la técnica del Grupo Focal (19), a través de ésta se realizaron preguntas estructuradas y directas con especificaciones de tipo abierto, claras, neutrales, ordenadas lógicamente, dirigidas hacia un único tema. En la tabla 2 se describen las categorías y subcategorías exploradas; tales como aspectos vinculados a la pareja, razones del rompimiento de la relación, duelo, y aspectos académicos.

\section{Categorización}

\begin{tabular}{|c|c|c|c|}
\hline $\begin{array}{l}\text { Categoria de } \\
\text { análizis }\end{array}$ & Definición conceptual & Definición de las subcategorias & Pregunta \\
\hline Pareja & $\begin{array}{l}\text { Las relaciones de pareja } \\
\text { corresponden a una de las } \\
\text { uniones más intensas que } \\
\text { se pueden establecer } \\
\text { entre los seres humanos, } \\
\text { y son el vínculo de mayor } \\
\text { significación fuera de la } \\
\text { familia de origen } \\
\text { QMaureira, 2011). }\end{array}$ & $\begin{array}{l}\text { Las relaciones amorosas fueron } \\
\text { una catezoria de analisis en la } \\
\text { que se exploró la concepción de } \\
\text { lo que significa tener pareja y lo } \\
\text { conlleva desde el punto personal } \\
\text { y subjetivo de cómo vive el } \\
\text { noviazgo cada participante } \\
\text { Es una categoria de analisis que } \\
\text { se describe con diferentes } \\
\text { acciones, emociones y } \\
\text { pensamientos de lo que es tener } \\
\text { pareja, por cada participante. }\end{array}$ & $\begin{array}{l}\text { 1. ¿Qué características } \\
\text { positivas debe tener una } \\
\text { relación de pareja desde tu } \\
\text { perspectiva? y } \text { Con qué } \\
\text { acciones o conductas se } \\
\text { pueden manifestar estas } \\
\text { caracteristicas? (p. ej. } \\
\text { respeto, tolerancia). }\end{array}$ \\
\hline $\begin{array}{l}\text { Razones para } \\
\text { el } \\
\text { Rompimiento }\end{array}$ & $\begin{array}{l}\text { Una ruptura puede ser } \\
\text { catalogada como un tipo } \\
\text { de duelo enigmitico } \\
\text { donde se presentan } \\
\text { emociones y conductas } \\
\text { negativas, de igual } \\
\text { manera se pueden } \\
\text { manifestar conflictos en } \\
\text { otras relaciones sociales } \\
\text { de la persona (Boss. } \\
2001 \text { ). }\end{array}$ & $\begin{array}{l}\text { Las razones del rompimiento } \\
\text { varian entre los participantes, } \\
\text { pero una constante es el estrés y } \\
\text { las emociones negativas que se } \\
\text { generó a partir de esta. } \\
\text { Debido a esto el contacto con la } \\
\text { expareja es minimo e inclusive } \\
\text { se rompió cualquier tipo de } \\
\text { comunicación para evitar que se } \\
\text { generen nuevamente una } \\
\text { desestabilidad. }\end{array}$ & $\begin{array}{l}\text { 2. En tu última relación } \\
\text { ¿Cuiles fueron las razones } \\
\text { por las cuales ésta } \\
\text { terminó? } \\
\text { 3. ¿Estuvieron de acuerdo } \\
\text { ambos con la ruptura? } \\
\text { 4. ¿Cómo es su relación } \\
\text { actualmente (amigos, } \\
\text { compañeros, colegas)? }\end{array}$ \\
\hline $\begin{array}{l}\text { Percepción } \\
\text { del Duelo }\end{array}$ & $\begin{array}{l}\text { El duelo es un proceso } \\
\text { que las personas } \\
\text { atraviesan tras la muerte } \\
\text { o perdida de algun ser } \\
\text { querido, sea familiar, } \\
\text { migo o pareja, es aqui } \\
\text { donde pasarán por una } \\
\text { serie de fases para la } \\
\text { superación de dicha } \\
\text { pérdida (Sanchez-Aragón } \\
\text { \& Martinez-Cruz, 2014). }\end{array}$ & $\begin{array}{l}\text { El duelo ante la ruptura amorosa } \\
\text { de los participantes se exploró } \\
\text { teniendo como referencia las } \\
\text { etapas del duelo de Kübler Ross } \\
\text { (1969). } \\
\text { Se exploró mediante el dialogo } \\
\text { con los participantes, } \\
\text { solicitandoles que expresaran las } \\
\text { emociones, sentimientos, } \\
\text { pensamientos y } \\
\text { comportamientos, asociados con } \\
\text { la percepción de la ruptura } \\
\text { amorosa. }\end{array}$ & $\begin{array}{l}\text { 5. ¿Cuiles freron los } \\
\text { sentimientos que se } \\
\text { presentaron al momento de } \\
\text { terminar (alegria, tristeza, } \\
\text { enojo)? CCómo se } \\
\text { manifestaron? } \\
\text { 6. ¿Estos sentimientos se } \\
\text { siguen presentando? } \\
\text { 7. A Aproximadamente cuanto } \\
\text { tiempo duraron estos } \\
\text { sentimientos? }\end{array}$ \\
\hline $\begin{array}{l}\text { Aspectos } \\
\text { académicos }\end{array}$ & $\begin{array}{l}\text { El rendimiento } \\
\text { académico es el nivel de } \\
\text { conocimiento demostrado } \\
\text { en un irea o materia } \\
\text { Torres \& Rodriguez, } \\
\text { 2006). El cual puede } \\
\text { verse afectado ya que } \\
\text { esta directamente } \\
\text { relacionado con factores } \\
\text { psicologicos como la } \\
\text { poca motivación, el } \\
\text { desinterés o las } \\
\text { distracciones, que } \\
\text { dificultan la comprensión } \\
\text { de los conocimientos } \\
\text { impartidos por el docente } \\
\text { y termina afectando al } \\
\text { rendimiento académico a } \\
\text { la hora de las } \\
\text { evaluaciones (Gardes. } \\
2008 \text { ). }\end{array}$ & $\begin{array}{l}\text { Los aspectos académicos se } \\
\text { exploraron a partir de datos } \\
\text { referidos por los participantes, } \\
\text { donde percibieron que han } \\
\text { disminuido su rendimiento } \\
\text { academico el cual entia asociado } \\
\text { con la ruptura amorosa, tomando } \\
\text { en cuenta los siguientes } \\
\text { elementos: } \\
\text {-Si existe una menor capacidad } \\
\text { de concentración } \\
\text {-Si existe existen menos ganas } \\
\text { de ir a la escuela } \\
\text {-Si hay un menor interés por las } \\
\text { clases } \\
\text {-Si hay pensamientos recurrentes } \\
\text { sobre su ruptura, que impida } \\
\text { realizar su vida académica. } \\
\end{array}$ & $\begin{array}{l}\text { 8. ¿Se vio afectada el area } \\
\text { académica con este } \\
\text { rompimiento? ¿De qué } \\
\text { forma? } \\
\text { 9. ¿Te sentias con la } \\
\text { misma motivación al realizrr } \\
\text { tus trabajos escolares? } \\
\text { 10. Dejabas de asistir a } \\
\text { clases para realizar una } \\
\text { actividad que ayudara a } \\
\text { distraerte (por causa del } \\
\text { rompimiento)? } \\
11 . \quad \text { Te concentrabas con } \\
\text { la misma facilidad en clases o } \\
\text { al realizar alguna tarea? } \\
12 \text { iTe sentias con la } \\
\text { misma energia para cumplir } \\
\text { con responsabilidades } \\
\text { escolares? }\end{array}$ \\
\hline
\end{tabular}

\section{Procedimiento}

Este Protocolo siguió las Normas Éticas y de Investigación consideradas en la Declaración de Helsinki (22), en cuanto a la investigación con seres humanos. Para captar a los participantes, se realizaron publicaciones que se compartieron en diferentes redes sociales invitando a estudiantes universitarios que hubieran atravesado por una ruptura amorosa. Al momento de contactarles (antes de llevar a cabo el grupo focal), se aseguraba que contaran con las características de inclusión (estudiante universitario y haber terminado una relación teniendo seis meses como máximo). Una vez reunidos, a través de la plataforma Zoom (debido a las normas de sanidad por la pandemia de Covid-19 no era posible realizarlo de forma presencial), se explicó a los participantes los objetivos de la investigación, aclarando que la información compartida consideraba la confidencial (se firmó un Consentimiento Informado, aplicado a través de un formulario de Google), así mismo, se explicó con detalle la dinámica de la entrevista grupal. Un coordinador guio la entrevista, realizando las preguntas directamente, y de manera voluntaria los participantes, expresaban sus respuestas. Al finalizar se otorgó el agradecimiento.

\section{Resultados}

A continuación se describen los resultados obtenidos de acuerdo con cada categoría de análisis (Tabla 2), es importante destacar que se realizó el análisis de contenido de las respuestas brindadas por los participantes, así como la obtención de la frecuencia de estas, considerando las respuestas semejantes que daban; es decir, si entre cinco y seis participantes daban una respuesta semejante se consideraba una frecuencia alta, entre tres y cuatro una frecuencia media, y por último se consideraba una frecuencia baja entre uno a dos participantes. Los resultados se describen inicialmente mostrando las preguntas que se dirigieron a los participantes (por categoría), posteriormente se pueden observar los principales argumentos que explicaron durante su discurso, y de esta manera se obtuvo la frecuencia. Los resultados se complementan retomando parte de los discursos de algunos de los testimonios de los participantes.

\section{a) Pareja}

Respecto a las primeras preguntas: ¿Qué características positivas debe tener una relación de pareja desde tu perspectiva? y ¿Con qué acciones o conductas se pueden manifestar estas características? (p. ej. respeto, tolerancia). Los participantes explicaron que tienen perspectivas sobre algunas características positivas que debe tener una relación, las cuales se aprecian en la tabla 3. 
Tabla 3.

\begin{tabular}{lcl}
$\begin{array}{l}\text { Caracteristicas de una relación. } \\
\text { Característica }\end{array}$ & Frecuencia & \multicolumn{1}{c}{ Ejemplo de testimonio } \\
\hline Respeto & Alta & $\begin{array}{l}\text { Participante 3: "El respeto y la paciencia van de la } \\
\text { mano" }\end{array}$ \\
\hline Comunicación & Media & $\begin{array}{l}\text { Participante 2: "Comunicación porque... bueno } \\
\text { problemas siempre va a haber en una relación, pero si } \\
\text { no saben cómo solucionarlo, no saben cómo expresar } \\
\text { que es lo que sienten no hay nada." }\end{array}$ \\
\hline Confianza & Media & $\begin{array}{l}\text { Participante 4: "si no confias en esa persona no vas a } \\
\text { estar como o tu tranquila y tampoco va a haber } \\
\text { nada." }\end{array}$ \\
\hline Paciencia & Media & $\begin{array}{l}\text { Participante 2: "Bueno yo creo que hay 3 pilares muy } \\
\text { importantes que son el respeto, la paciencia y la } \\
\text { comunicación." }\end{array}$ \\
\hline Apoyo & Baja & $\begin{array}{l}\text { Participante 3: "Es importante que se apoyen entre sí } \\
\text { para que los dos puedan sentirse motivado para lograr } \\
\text { sus objetivos." }\end{array}$ \\
\hline Cariño & Baja & $\begin{array}{l}\text { Participante 5: "Al igual que es importante que se } \\
\text { apoyen y se demulestren cariño sino para que estén en } \\
\text { esa relación." }\end{array}$ \\
\hline
\end{tabular}

Como puede observarse en la tabla 3 , las características de una relación arrojaron datos asociados con una frecuencia alta al respeto, siguiéndole con frecuencia media: comunicación, confianza y paciencia, y con menor frecuencia: apoyo y cariño.

Tabla 4.

Aspectos positivos de las caracteristicas de la pareja.

\begin{tabular}{lcl}
\hline \multicolumn{1}{c}{ Conducta } & Frecuencia & \multicolumn{1}{c}{ Testimonio } \\
\hline $\begin{array}{l}\text { Respetar el } \\
\text { espacio del otro }\end{array}$ & Alta & $\begin{array}{l}\text { Participante 1: "Cada quien pueda tener su espacio, } \\
\text { sutiempo, sus amigos, todo, todo tranquilo," }\end{array}$ \\
\hline $\begin{array}{l}\text { Hablar sobre } \\
\text { posibles } \\
\text { problemas }\end{array}$ & Media & $\begin{array}{l}\text { Participante 5: "Alguien que este se sepa } \\
\text { comumicar, que sea muy abierto en cuamto a si se } \\
\text { siente cómodo, incómodo." }\end{array}$ \\
\hline Divertido & Baja & $\begin{array}{l}\text { Participante 3: "Que sea divertido, te haga reir, te } \\
\text { procure más que nada." }\end{array}$ \\
\hline Actitud positiva & Baja & $\begin{array}{l}\text { Participante 4: "Me fijo este por ejemplo en que } \\
\text { tenga una actitud como muy positiva, que no si tiene } \\
\text { como que algin problema, como que no se caiga y } \\
\text { que siempre busque como soluciones." }\end{array}$ \\
\hline Establecer metas & Baja & $\begin{array}{l}\text { Participante 6: "Que sepan un poco lo que quiereno } \\
\text { que tengan claras como que sus metas, sus } \\
\text { convicciones." }\end{array}$ \\
\hline
\end{tabular}

En la Tabla 4 se describen los aspectos positivos de las características de la pareja, nuevamente aparece con una frecuencia alta el respeto, pero ahora dirigido al espacio del otro; con frecuencia media el hablar de posibles problemas, y con frecuencia baja divertido, actitud positiva y establecer metas.

\section{b) Razones para el Rompimiento}

Respecto a la categoría "Razones para el rompimiento", se realizaron preguntas, a continuación se presentan los resultados que arrojó cada una de estas.

En cuanto a las preguntas sobre la última relación, tales como: ¿Cuáles fueron las razones por las cuales ésta terminó? Las respuestas se observan en la tabla 5.
Tabla 5

\begin{tabular}{|c|c|c|}
\hline Motivo & Frecuencia & Testimonio \\
\hline $\begin{array}{l}\text { Falta de } \\
\text { comunicación }\end{array}$ & Media & $\begin{array}{l}\text { Participante 5: "Simplemente si hubo como que un } \\
\text { comentario que no me agrado de él, yo pues le dije que no } \\
\text { me agrado, como que se molestó y ya pues asi un fin de } \\
\text { semana dejo de hablarme." }\end{array}$ \\
\hline $\begin{array}{l}\text { Aparición de } \\
\text { terceros }\end{array}$ & Media & $\begin{array}{l}\text { Participante 6: "Vi que subió a Facebook una foto con su } \\
\text { emovia, entonces pues no sé, creo que el momento si me } \\
\text { costó asimilarlo." }\end{array}$ \\
\hline Peleas & Baja & $\begin{array}{l}\text { Participante 3: "Empezamos a tener problemas porque ella } \\
\text { a fuerza queria estar en todos lados conmigo, yo no soy } \\
\text { mucho asi como de salir pero comencé a salir a inicio de } \\
\text { años con mis amigos y ella se molestaba porque no la } \\
\text { llevaba" }\end{array}$ \\
\hline Desconfianza & Baja & $\begin{array}{l}\text { Participante 4: "La desconfianza empezó a originar peleas } \\
\text { y peleas y más peleas, pero ya cada vez eran como que más } \\
\text { fuertes." }\end{array}$ \\
\hline Desinterés & Baja & $\begin{array}{l}\text { Participante 1: "Ehmm entonces pues nunca pensamos } \\
\text { realmente que ya no nos ibamos a ver y pues hubo un } \\
\text { momento donde pues dejamos de mostrar interés." }\end{array}$ \\
\hline Otros & Media & $\begin{array}{l}\text { Participante 2: "Pero él era muy inestable entonces un dia } \\
\text { asi de la nada estábamos súper bien y al dia siguiente me } \\
\text { dice no ya no quiero estar contigo." }\end{array}$ \\
\hline
\end{tabular}

Por otro lado, en la tabla 5 se describen las razones del rompimiento, en esta categoría con una frecuencia media se manifestó la falta de comunicación, la aparición de terceros (infidelidad), y otros aspectos tales como la inestabilidad. Con frecuencia baja se considero a las peleas, la desconfianza y el desinterés.

Respecto a preguntas que precisan aspectos de la relación, tales como: ¿Estuvieron de acuerdo ambos con la ruptura? ¿Cómo es su relación actualmente (amigos, compañeros, colegas)? , los resultados se observan en la Tabla 6.

\begin{tabular}{|c|c|c|c|c|}
\hline $\begin{array}{l}\text { Acordaron } \\
\text { la ruptura }\end{array}$ & Frecuencia & $\begin{array}{c}\text { Siguen } \\
\text { interactuando }\end{array}$ & Frecuencia & Testimonio \\
\hline Acuerdo & Media & $\begin{array}{l}\text { Ninguin } \\
\text { contacto }\end{array}$ & Media & 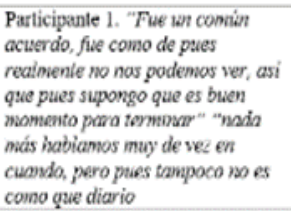 \\
\hline Desacuerdo & Media & $\begin{array}{l}\text { Minimo } \\
\text { contacto }\end{array}$ & Baja & 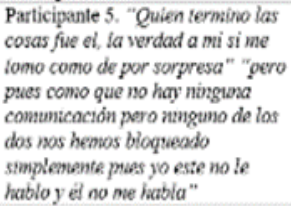 \\
\hline
\end{tabular}

En la tabla 6 se presentan aspectos relativos a la situación de ruptura y la posibilidad de contacto con la expareja, cabe destacar, que en las respuestas se dividieron de manera claramente definida con una frecuencia media que ambos acordaron la ruptura sin continuar la interacción entre ellos; y de forma semejante el no haber acordado la ruptura, teniendo entre ellos un mínimo contacto.

c) Percepción de duelo

Respecto a la categoría "Percepción del Duelo", se realizaron preguntas, en la Tabla 7 se presentan los resultados que arrojó cada una de estas.

Preguntas: - ¿Cuáles fueron los sentimientos que se presentaron al momento de terminar (alegría, tristeza, 
enojo)? ¿Cómo se manifestaron? ¿Estos sentimientos se siguen presentando? ¿Aproximadamente cuánto tiempo duraron estos sentimientos?

\begin{tabular}{|c|c|c|c|c|}
\hline Sentimientos & Frecuencia & Manifestación & Tiempo & Testimonio \\
\hline $\begin{array}{l}\text { Positivos } \\
\text { (tranquilidad, } \\
\text { paz) }\end{array}$ & Baja & Mejor concentración & & $\begin{array}{l}\text { Participante } 1: \text { "Ya } \\
\text { ahorita me centre en lo } \\
\text { que quiero hacer y en } \\
\text { mis planes" }\end{array}$ \\
\hline $\begin{array}{l}\text { Negativos } \\
\text { (enojo, } \\
\text { tristeza) }\end{array}$ & Alta & $\begin{array}{l}\text { Llanto, alteraciones } \\
\text { en la ingesta de } \\
\text { alimentos y de sueño }\end{array}$ & $\begin{array}{l}\text { De tres } \\
\text { a cuatro } \\
\text { meses }\end{array}$ & $\begin{array}{l}\text { Participante 4: "Dormia } \\
\text { más de lo debido o sea } \\
\text { cómo que era que me } \\
\text { despertaban y ¿quieres } \\
\text { comer? no quiero y me } \\
\text { volvi a dormir" }\end{array}$ \\
\hline
\end{tabular}

En cuanto a la percepción del proceso de duelo por la ruptura amorosa, se obtuvieron respuestas con frecuencia alta vinculadas a sentimientos negativos como enojo y tristeza, que incluyen llanto, alteraciones en la ingesta de alimentos y problemas con el sueño; paradójicamente con una frecuencia baja se comentó que la ruptura trajo sentimientos positivos asociados con tranquilidad y paz, así como mejor concentración.

Por otro lado, como uno de los objetivos de este estudio se analizaron las etapas del duelo respecto a la ruptura amorosa, en este aspecto también se ejemplifica el dato a través del discurso de los participantes. De manera general, se puede señalar que no se observaron las etapas de manera secuencial, sino más bien de forma indistinta, pero claramente diferenciadas como: negación, ira, negociación, depresión y aceptación.

\begin{tabular}{|c|c|}
\hline Etxpa & Testimonio \\
\hline Negactóa & 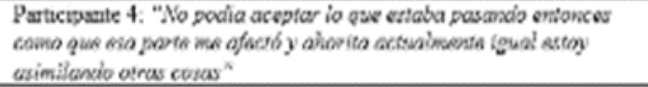 \\
\hline Ina & 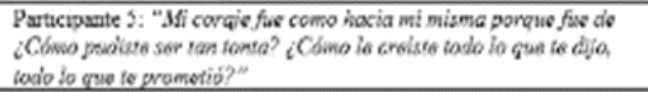 \\
\hline Negociscića & 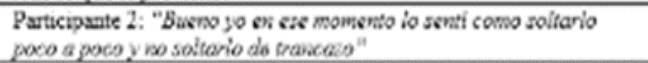 \\
\hline Depersón & 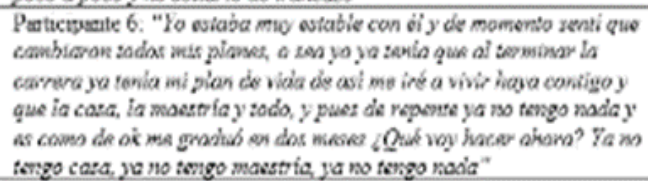 \\
\hline Acepteción & 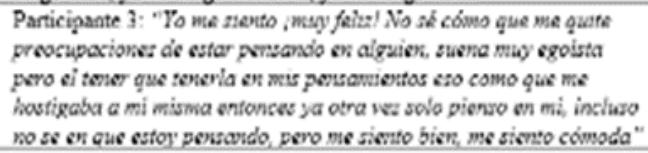 \\
\hline
\end{tabular}

\section{d) Aspectos académicos}

Respecto a la categoría "Aspectos académicos", se tomaron en cuenta elementos de acuerdo con el discurso de los participantes que pudieran haberse afectado a partir de la ruptura amorosa, entre los señalados con una frecuencia media se presentaron: bajo promedio, materias reprobadas, incumplimiento en trabajos o con poca calidad. La falta de motivación asociada con falta de ánimo y pensamientos negativos. Si bien no se reportó el realizar otras actividades para "distraerse", si se verbalizaron aspectos como: falta de noción del tiempo, no recordar las clases, preferir dormir $u$ otras actividades. También se manifestó la falta de concentración en las clases debido a pensamientos asociados con el rompimiento. Se presentaban también sensaciones de cansancio y falta de ánimos, como contrarias a la energía para cumplir con tareas y clases. Las respuestas se observan en la tabla 9. Tabla 9.

\begin{tabular}{|c|c|c|}
\hline Pregunta & Caracteristicas Frccucncia & Testimonio \\
\hline $\begin{array}{l}\text { ¿Se vio afectada } \\
\text { cl arca académica } \\
\text { coa este } \\
\text { rompiniento? } \\
\text { ¿De qué forma? }\end{array}$ & $\begin{array}{l}\text { Bajo promedio. Media. } \\
\text { materias } \\
\text { reprobadas, } \\
\text { incumpliniento } \\
\text { con trabajos o } \\
\text { con poxa } \\
\text { calidad. }\end{array}$ & $\begin{array}{l}\text { Participante 1: "Si habia materias } \\
\text { que reprobe entonces si mfingo en } \\
\text { wi semestre, perdi un semestrey no } \\
\text { es cómo echarie ia culpa a mi ex } \\
\text { novia pero pues reaimente ingluyb } \\
\text { todo esto que teria." }\end{array}$ \\
\hline $\begin{array}{l}\text { ¿Te sentias cou la } \\
\text { misma } \\
\text { motivación al } \\
\text { realizar tus } \\
\text { trabajos } \\
\text { escolares? }\end{array}$ & $\begin{array}{l}\text { Falta de ánimos, Media. } \\
\text { pensannicntos } \\
\text { uegativos. }\end{array}$ & $\begin{array}{l}\text { Participante 2: "No tenia ganas de } \\
\text { nada, es que cra muy extraio te } \\
\text { digo porque no me seatia súper } \\
\text { derrotada." }\end{array}$ \\
\hline $\begin{array}{l}\text { Dejabas de } \\
\text { asistir a clases } \\
\text { para realizar una } \\
\text { actividad que } \\
\text { ayudará a } \\
\text { distraerte (por } \\
\text { causa del } \\
\text { rompimiento)? }\end{array}$ & $\begin{array}{l}\text { Falta de nocióa Modia. } \\
\text { del tiempo, no } \\
\text { rocordar las } \\
\text { clases, preferir } \\
\text { dormir u otras } \\
\text { actividades. }\end{array}$ & $\begin{array}{l}\text { Participante 4: "Me sentia cansada } \\
\text { ilegue a faltar a algunas clases por } \\
\text { quedame dormida o se me iba el } \\
\text { nempo swn damme cuents." }\end{array}$ \\
\hline $\begin{array}{l}\text { Te concentrabas } \\
\text { coa la misma } \\
\text { facilidad en clases } \\
\text { o al realizar } \\
\text { alguna tarca? }\end{array}$ & $\begin{array}{l}\text { Pensar en el Media. } \\
\text { rorupimiento. }\end{array}$ & $\begin{array}{l}\text { Participante 5: "para mi es mucho } \\
\text { esos pensamienios es que siempre } \\
\text { estoy pensando, pero por qué por } \\
\text { qué terminó porque me dejo." }\end{array}$ \\
\hline $\begin{array}{l}\text { iTe sentias con la } \\
\text { misma energia } \\
\text { para cumplir coa } \\
\text { responsabilidades } \\
\text { escolares? }\end{array}$ & $\begin{array}{l}\text { Sensacióa de Media. } \\
\text { cansancio, falta } \\
\text { de ánimos. }\end{array}$ & $\begin{array}{l}\text { Participante 6: "se me iba la onda } \\
\text { yo me la pasaba o dormida o } \\
\text { llorando todo el dia, cntonces } \\
\text { cuando menos vi, ya tengo la tesis } \\
\text { cancelada }\end{array}$ \\
\hline
\end{tabular}

\section{Discusión}

Se puede afirmar que el objetivo de esta investigación se alcanzó, ya que sí se encontraron elementos vinculados entre las categorías y subcategorías de análisis exploradas, y se observó en estudiantes universitarios cómo se manifiesta la ruptura amorosa en la relación de pareja y su percepción del proceso de duelo, del mismo modo se analizó la relación con aspectos académicos.

Boss ${ }^{23}$ señala que la ruptura amorosa en la pareja suele presentar un tipo de duelo que se manifiesta de manera ambigua, que es muy común en la vida de las personas, donde se experimentan estados afectivos y cognoscitivos negativos, que incluyen la afectación de las relaciones en el entorno social del individuo, así como conflictos en las relaciones sociales del entorno íntimo de la persona. En este sentido, los resultados de este reporte confirman lo señalado por Boss, así como por González ${ }^{8}$, ya que en la percepción de los participantes del proceso de duelo, se manifestaron respuestas vinculadas con sentimientos negativos, tales como enojo y tristeza, agregado con llanto (este último también lo describen Keller y Nesse ${ }^{24}$, como un modo "adaptativo" al problema emocional que se está 
enfrentando); así mismo, se presentan problemas de salud física, entre los que destacan las alteraciones en la ingesta de alimentos y problemas con el sueño.

Paradójicamente, los participantes de nuestro estudio también comentaron que la ruptura trajo consigo sentimientos positivos asociados con "tranquilidad y paz", así como "mejor concentración", esto también se confirma con lo señalado por Clapp ${ }^{18}$ y Bustos Caro ${ }^{25}$, quienes describen efectos constructivos para las personas que han padecido el rompimiento de pareja, y una vez que lo superan logran nuevos significados que favorecen el crecimiento personal, y afrontan nuevos retos con mayor seguridad.

Ahora bien, en cuanto a las principales razones por las cuales la relación de noviazgo finalizó, los participantes señalaron a la falta de comunicación, así como la aparición de "terceros" (infidelidad), además de peleas, desconfianza, y desinterés; estos datos son semejantes a lo señalado por Córdova ${ }^{26}$, quien encontró en su estudio que entre las razones asociadas para terminar la relación se presentan la desconexión y desinterés, el no cumplir con las expectativas, la sensación de "no va a cambiar", la aparición de terceros y la desconfianza. Así mismo, Barajas $^{12}$, describen sentimientos y emociones negativos, que incluyen tristeza, dolor, enojo, engaños e infidelidad. En cuanto a la percepción del proceso de duelo por la ruptura amorosa, los datos recabados en este estudio confirmaron de alguna manera las etapas definidas teóricamente por diferentes autores ${ }^{7}$, en nuestro estudio no se identificaron etapas de manera secuencial, sino más bien de forma indistinta, pero claramente diferenciadas por discursos asociados con la negación, ira/hostilidad, depresión, aceptación, negociación, desesperanza.

Por otro lado, en cuanto a lo observado en el tema de los aspectos académicos asociados a la ruptura amorosa, se encontró que se presentaron: bajo promedio, materias reprobadas, incumplimiento en trabajos o con poca calidad, falta de motivación asociada con falta de ánimo y pensamientos negativos. Si bien no se reportó el realizar otras actividades para "distraerse", si se verbalizaron aspectos como: problemas en la noción del tiempo, no recordar las clases, preferir dormir a realizar actividades escolares. También se manifestó la falta de concentración en las clases debido a pensamientos asociados con el rompimiento. Se presentaban también sensaciones de cansancio y falta de ánimos, como contrarias a la energía para cumplir con tareas y clases. De alguna forma los resultados concuerdan con lo señalado por De Martínez Gómez ${ }^{27}$, quienes describen que como parte de las etapas del duelo aparece de manera importante el desequilibrio emocional que impacta en diferentes áreas de la vida de quien lo padece, caracterizado por sentimientos negativos asociados a la desesperanza, y que principalmente impide continuar con la rutina diaria.
Particularmente la vida cotidiana de los jóvenes estudiantes se afecta de manera importante, como lo señalan sus testimonios; lo mismo encontraron Medina ${ }^{13}$, Pulido ${ }^{14}$, Morán ${ }^{15}$, quienes identificaron reprobación, baja en calificaciones, falta de motivación para continuar con los estudios, falta de concentración, aunado a problemas físicos. El área escolar de los jóvenes universitarios se vio también afectada por diferentes causas, que de acuerdo con los testimonios implicó: bajo promedio, materias reprobadas, incumplimiento de trabajos o con poca calidad, pensar continuamente en el rompimiento.

De manera general se puede afirmar que los resultados obtenidos en este estudio, tienen concordancia con reportes de investigación previos, que señalan que las emociones, pensamientos y conductas que experimentan las personas jóvenes durante una ruptura de pareja, pueden desencadenar sintomatología asociada a la depresión, ansiedad, hostilidad y problemas somáticos 28; que afecta diversas áreas de la vida cotidiana, incluyendo el área escolar, que de manera importante tendrá consecuencias asociadas con la reprobación.

Por lo tanto, es importante intervenir oportunamente durante el proceso de duelo por la ruptura amorosa para evitar trascender a cuadros que afecten de manera importante la salud mental de los jóvenes. Las pérdidas afectivas que se dan en el contexto personal de los individuos representa un reto para quien las vive, pero también para los profesionales de la salud mental que brindan atención a los estudiantes en el ámbito escolar. La investigación publicada y actual es relativamente escasa, considerando la relación entre la ruptura amorosa, el proceso de duelo y factores académicos, datos como los recabados en este estudio pueden ser el punto de partida para un estudio profundo que incluya establecer diferencias por sexo, considerar el tiempo de ruptura de la relación entre otros; así como implementar estrategias que puedan fortalecer al individuo para seguir adelante, y afrontar de manera saludable la pérdida afectiva.

Ante los resultados de esta investigación se sugiere desarrollar en las instituciones educativas intervenciones y grupos de apoyo para jóvenes que pueden verse afectados ante esta situación. Las consecuencias que desencadena un proceso de duelo pueden variar al igual que la intensidad con la que se presentan, por lo tanto, es recomendable estar preparado ante cualquier situación brindando contención emocional, fortalecer la red de apoyo social, entre otras.

\section{Referencias}

[1] Maureira F. Los cuatro componentes de la relación de pareja. Revista Electrónica de Psicología Iztacala. 2011; 14 (1): 321-331. http://revistas.unam.mx/index.php/repi/article/viewFile/24815/23284 
[2] Becerril D. Relaciones de pareja. Revista de Educación. 2001; 325: 4956. http://www.educacionyfp.gob.es/dam/jcr:d62d42d8-cae9-4d7d8a67-024aba99e763/re3250509962-pdf.pdf

[3] García FE, Ilabaca Martínez D. Ruptura de pareja, afrontamiento y bienestar psicológico en adultos jóvenes. Ajayu. 2013; 11 (2): 157-172. https://www.researchgate.net/profile/Felipe_Garcia14/publication/317 528981_RUPTURA_DE_PAREJA_AFRONTAMIENTO_Y_BIENE STAR_PSICOLOGICO_EN_ADULTOS_JOVENES/links/597137fea ca2721b09790fee/RUPTURA-DE-PAREJA-AFRONTAMIENTO-YBIENESTAR-PSICOLOGICO-EN-ADULTOS-JOVENES.pdf

[4] Barajas M, Cruz del Castillo C. Ruptura de la pareja en jóvenes: factores relacionados con su impacto. Enseñanza e investigación en Psicología. 2017; 22(3): 342-352. https://www.redalyc.org/articulo.oa?id=29255775008

[5] Zurita G. Qué es el duelo. Las etapas del duelo. Instituto Galene. 2020. https://galene.es/duelo/que-es-el-duelo-las-etapas-del-duelo

[6] Oviedo Soto SJ, Parra Falcón M, Marquina Volcanes M. La muerte y el duelo. Enfermería Global. 2009; (15), 1-9. http://scielo.isciii.es/scielo.php?script=sci_arttext\&pid=S1695$61412009000100015 \& \operatorname{lng}=$ es\&tlng=es

[7] Galán M. MODELO DE LAS 5 FASES DE ELISABETH KÜBLERROSS. Psicologa Cáceres. 2017. https://cacerespsicologo.com/losmodelos-de-adaptacion-a-la-enfermedad-terminal-por-elisabehtkubler-ross/

[8] González F. Narrativas de la experiencia de mujeres mexicanas de la ciudad de Guadalajara Jalisco en el proceso de duelo por divorcio. Revista de Psicoterapia Relaciones e Intervenciones Sociales. 2016; 34 47- 40

[9] Diaz Loving R, Sánchez Aragón R. Psicología del amor: Una visión integral de la relación de pareja. México: Porrúa; 2004

[10] Vargas JL. 6 razones por las que se debe terminar las relaciones de pareja. Biobiochile. 2016 https://www.biobiochile.cl/noticias/2016/04/10/6-razones-por-lasque-se-terminan-las-relaciones-de-pareja.shtml

[11] Sánchez AR, Martínez CR. Causas y caracterización de las etapas del duelo romántico. Acta de Investigación Psicológica. 2014; 4(1): 1329. 1343.

https://reader.elsevier.com/reader/sd/pii/S2007471914703783?token= 097374E514474E1F490D506770DE208C920A21C01F765FE206481 89615C2DE845D89CAA9958D1EA8CEB4AEB55824E7EB

[12] Barajas M, González C, Cruz C, Robles R. El significado psicológico de una ruptura de pareja significativa en jóvenes universitarios Psicología Iberoamericana. 2012; 20(2): 26-32. https://www.researchgate.net/publication/319532242_El_significado_ psicologico_de_una_ruptura_de_pareja_significativa_en_jovenes_uni versitarios.

[13] Medina N, Fereira J, Marzol R. Factores personales que inciden en el bajo rendimiento académico de los estudiantes de geometría. Revista de Estudios Interdisciplinarios en Ciencias Sociales. 2018; 20 (1):4-28. https://dialnet.unirioja.es/descarga/articulo/6436353.pdf

[14] Pulido E, Lubo M. Creencias y significados atribuidos a la ruptura de pareja y su afrontamiento de jóvenes universitarios. [Tesis de Licenciatura]. Colombia: Fundación Universitaria Católica Lumen Gentium; 2018 https://repository.unicatolica.edu.co/bitstream/handle/20.500.12237/1 325/CREENCIAS_SIGNIFICADOS_ATRIBUIDOS_RUPTURA_P AREJA_AFRONTAMIENTO_JOVENES_UNIVERSITARIOS.pdf? sequence $=1 \&$ is Allowed $=\mathrm{y}$

[15] Moran Ayala LP. La depresión ante el desamor en jóvenes de 20 a 25 años en la Universidad Salesiana México. Revista VITAM. 2020. http://www.revistavitam.mx/unisal/intellectus/la-depresion-ante-eldesamor-en-jovenes-de-20-a-25-anos-de-edad-en-la-universidadsalesiana-mexico/
[16] Alvarado M, Gómez M. El noviazgo como un factor que influye en el éxito o fracaso escolar del adolescente [Tesis inédita de licenciatura]. México: Universidad Pedagógica Nacional; 2012. http://200.23.113.51/pdf/29008.pdf

[17] Martínez-Sánchez A, Hernández-Arzola LI, Carrillo-Méndez D, Romualdo-Pérez Z, Hernández-Miguel C. Factores asociados a la reprobación estudiantil en la Universidad de la Sierra Sur, Oaxaca Temas de Ciencia y Tecnología. 2013;17 (51): 25-33. http://www.utm.mx/edi_anteriores/temas51/T51_1Ensayo3FactAsocReprobacion.pdf

[18] Clapp G. Divorce and New Beggings. New York: Wiley; 2000

[19] Martínez N R. Reseña metodológica sobre los grupos focales. $\begin{array}{llll}\text { Diálogos. } & 2012 ; & 9, & \text { 47-53. }\end{array}$ https://core.ac.uk/download/pdf/47265053.pdf

[20] Torres LE, Rodríguez NY. Rendimiento académico y contexto familiar en estudiantes universitarios. Enseñanza e investigación en Psicología. 2006; $\quad 11 \quad$ (02), 255-270. https://www.redalyc.org/pdf/292/29211204.pdf

[21] Gardey A. Definición de rendimiento académico. Definición.de. 2008. https://definicion.de/rendimiento-academico/

[22] Asociación Médica Mundial. Declaración de Helsinki. Principios éticos para las investigaciones médicas en seres humanos $\left(59^{\mathrm{a}}\right.$ Asamblea General, Seúl, Corea, octubre 2008). Punto 32. http://www.ctomedicina.com/impugnaciones2014/bibliografiaP202MI R.pdf

[23] Boss P. (2009). The Trauma and Complicated Grief of Ambiguous Loss. Pastoral Psychology. 2009; 59 (2): 137-145.

[24] Keller M, Nesse R. Is low mood an adaptation? Evidence for subtypes with symptoms that match precipitants. Journal of Affective Disorders. 2005; 86 (1):27- 35 .

[25] Bustos Caro A. Cuando se acaba el amor: Estrategias de Afrontamiento, Duelo por Pérdidas Amorosas y Crecimiento Postraumático en estudiantes universitarios [Tesis inédita de licenciatura] Ecuador: Universidad San Francisco de Quito; 2011. https://docplayer.es/11403523-Universidad-san-francisco-de-quitocolegio-de-artes-liberales.html

[26] Alzugaray C, García F. La felicidad de los chilenos: Estudios sobre bienestar. Relaciones de pareja y bienestar psicológico. 2015; 1:237251

https://www.researchgate.net/publication/294891178_Relaciones_de_ pareja_y_bienestar_psicologico

[27] Solano Luengo LO. Rendimiento académico de los estudiantes de secundaria obligatoria y su relación con las aptitudes mentales y las actitudes ante el estudio [Tesis inédita doctoral]. Departamento de Métodos de Investigación y Diagnóstico en Educación II. 2015. http://e-spacio.uned.es/fez/eserv/tesisuned:EducacionLosolano/SOLANO_LUENGO_Luis_Octavio.pdf

[28] García DF. Narración del duelo en la ruptura amorosa. Ajayu. 2014; 12 (2): 288-307. https://www.redalyc.org/pdf/4615/461545458003.pdf 